\title{
Die Kodifizierung von Gestaltungswissen in interdisziplinären Entwurfsmustern
}

\author{
Lösungen im Spannungsfeld zwischen \\ Rechtsverträglichkeit und Dienstleistungsqualität
}

In der Entwicklung informationstechnischer Systeme entstehen oft Zielkonflikte, die durch Gestaltung der Systeme aufgelöst werden müssen. Hierfür fehlt es Entwicklern, insbesondere bei Konflikten zwischen Rechtsverträglichkeit und Dienstleistungsqualität, oft an dem notwendigen Wissen und der erforderlichen Gestaltungserfahrung. Hier können Entwurfsmuster weiterhelfen. Der Beitrag stellt am Beispiel Smarter Persönlicher Assistenten einen Ansatz vor, in dem Entwurfsmuster interdisziplinäres Gestaltungswissen zusammenführen, geht auf die Besonderheiten des Gestaltungswissens der beiden Disziplinen ein und stellt ein beispielhaftes Entwurfsmuster vor.

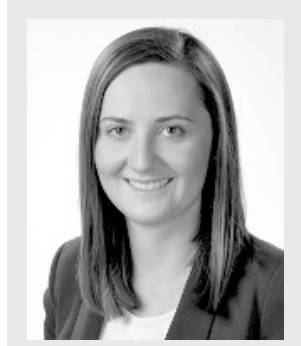

\section{Ernestine Dickhaut, M.Sc.}

Doktorandin und Wissenschaftliche Mitarbeiterin am Fachgebiet für Wirtschaftsinformatik und dem Wissenschaftlichen Zentrum für Informationstechnikgestaltung (ITeG) an der Universität Kassel.

E-Mail: ernestine.dickhaut@uni-kassel.de

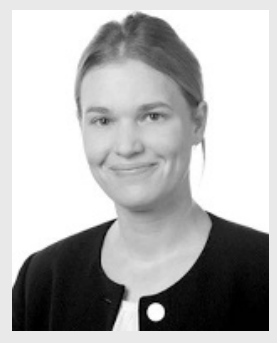

Laura Friederike Thies, MLE.

Wissenschaftliche Mitarbeiterin in der Projektgruppe verfassungsverträgliche Technikgestaltung (provet) im Wissenschaftlichen Zentrum für InformationstechnikGestaltung (ITeG) der Universität Kassel.

E-Mail: I.thies@uni-kassel.de

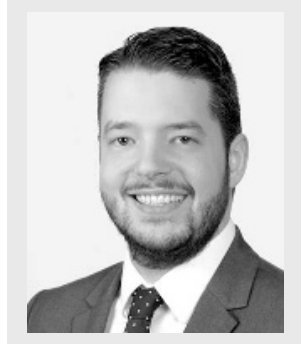

\section{Dr. Andreas Janson}

Forschungsgruppenleiter und Postdoktorand am Fachgebiet für Wirtschaftsinformatik und dem Wissenschaftlichen Zentrum für Informationstechnikgestaltung (ITeG) an der Universität Kassel. E-Mail: andreas.janson@uni-kassel.de

\section{Anforderungskonflikte}

In der Entwicklung datenschutzgerechter Systeme fehlt es Entwicklern häufig an rechtlichem Wissen und dem notwendigen Verständnis, die rechtlichen Anforderungen zufriedenstellend umzusetzen. Das führt dazu, dass rechtlichen Aspekten häufig erst zum Ende der Entwicklung Beachtung geschenkt wird. Und meist auch nur so weit, dass diese gerade so den Mindestanforderungen - damit das System ohne Sanktionsrisiko auf den Markt gebracht werden darf - entsprechen.

Neben mangelndem Rechtswissen sind Konflikte mit anderen Gestaltungskriterien, wie der Dienstleistungsqualität, eine Herausforderung. So ist etwa die Personalisierung von Systemen, insbesondere von Smarten Persönlichen Assistenten (SPA), ein entscheidendes Merkmal für die Benutzerfreundlichkeit und damit der Dienstleistungsqualität. Dagegen fordert das Datenschutzrecht, so wenig personenbezogene Daten wie möglich zu verarbeiten. Solche Konflikte sollen durch Systemgestaltung gelöst werden. Um die Gestaltungsvorschläge in die Systementwicklung einzuführen, sind Entwurfsmuster ein geeignetes Instrument.

Um den Anforderungen von Recht und Dienstleistungsqualität gerecht zu werden, erläutert der Beitrag die Unterschiede in der Kodifizierung von Rechts- und Dienstleistungswissen und stellt einen interdisziplinären Ansatz vor, der beide Disziplinen vereint. Dabei werden die beiden unterschiedlichen Arten der Wissenskodifizierung im Recht und in der Technik in gemeinsamen Entwurfsmustern verknüpft.

* Beitrag zum DFG-Forschungsprojekt "Anforderungs- und Entwurfsmuster zur rechtsverträglichen und qualitätszentrierten Entwicklung kontextsensitiver Applikationen" (AnEkA), https://www.uni-kassel.de/fb07/institute/iwr/personenfachgebiete/rossnagel-prof-dr/forschung/provet/aneka.html. 


\section{Entwurfsmuster}

In der Regel startet die Systementwicklung mit einer Anforderungserhebung. In der Anforderungserhebung werden konkrete Vorgaben für das Endprodukt festgehalten, um darauf aufbauend die Technologie zu entwickeln. Die Anforderungen werden hier meist in Gesprächen und schriftlichen Austausch gesammelt und für die weitere Verwendung in der Systementwicklung kodifiziert - eventuell in Anforderungsmustern. ${ }^{1}$ Die Basis des kodifizierten Wissens stellt die natürliche Sprache dar. Jedoch können die erhobenen Anforderungen durch einen Entwickler ${ }^{2}$ nicht lediglich in natürlicher Sprache in das Programm integriert werden. Die Anforderungen müssen durch den Entwickler in den Programmcode der verwendeten Programmiersprache transferiert und zu einem Gesamtprogramm erstellt werden. Dies stellt den Entwickler vor diverse Herausforderungen. Da es sich häufig um Probleme handelt, die immer wieder auftreten, ist es unter Entwicklern üblich, sich über auftretende Probleme auszutauschen. Neben dem Einsatz von sogenannten Dokumentationen, in denen bestimmte Regeln für die Programmiersprache festgehalten werden, nutzen viele Entwickler Online-Austauschforen. Bekannte Plattformen sind beispielsweise stackoverflow. com und java.forum.org. ${ }^{3}$ Der Austausch auf diesen Plattformen läuft häufig nach folgendem Schema ab: Es wird eine Frage gestellt, in der das Problem kurz in natürlicher Sprache erklärt wird, es werden technische Details, die verwendete Programmiersprache und andere Bedingungen angegeben und ein Ausschnitt des Programmcodes eingefügt. Auch die Antworten auf die gestellten Fragen ähneln sich in ihrem Aufbau sehr. Sie enthalten in den meisten Fällen kurze Ausschnitte von Programmcode, die das Problem lösen sollen.

Gestaltungswissen ist eine besondere Form von Wissen, nämlich das Wissen, ein System einschließlich Methoden und Konstruktionen zu entwerfen. ${ }^{4} \mathrm{Da}$ Wissen oft vage formuliert ist, erfordert die Kodifizierung von Gestaltungswissen spezielle Methodiken. Es gibt bereits verschiedene Ansätze, die sich mit Methoden zur Kodifizierung von Gestaltungswissen in der Systementwicklung beschäftigt haben, um das Wissen für die Zukunft zu erfassen und an andere weiterzugeben. Bekannte Formen für die Kodifizierung von Wissen sind beispielsweise Mind maps ${ }^{5}$, Cheat Sheets ${ }^{6}$, Wikis ${ }^{7}$, Gestaltungsprinzipien ${ }^{8}$ und Entwurfsmuster ${ }^{9}$. In der Praxis haben sich insbesondere Entwurfsmuster durchgesetzt, um Gestaltungswissen für die Zukunft zu kodifizieren und dadurch nutzbar zu machen. Entwurfsmuster bieten Lösungsansätze für immer wieder auftretende Probleme. ${ }^{10}$ Sie werden in der Regel tabellarisch dargestellt. ${ }^{11}$ Die dargestellten Muster bie-

1 Siehe Dickhaut/Janson/Roßnagel/Leimeister, in diesem Heft

2 Zur besseren Lesbarkeit des Textes wird auf die Aufzählung mehrerer Geschlechter verzichtet. Die Begriffe "Entwickler" oder "Nutzer" und ähnliche Begriffe umfassen immer auch alle Personen anderen Geschlechts.

3 Abtahi/Dietz, Learning Rust: How Experienced Programmers Leverage Resources to Learn a New Programming Language, 1.

4 Gregor/Hevner, MIS quarterly 2013.

5 Wheeldon/Faubert, International Journal of Qualitative Methods 8, 2009, 68.

6 Song/Guo/Thuente, IEEE Frontiers in Education 2016, 1.

7 Phuwanartnurak, Interdisciplinary Collaboration through Wikis in Software Development., 2009.

8 Seidel et al., European Journal of Information Systems 27 (2018), 221.

9 Alexander, The timeless way of building, 1979.

10 Alexander, A pattern language: towns, buildings, construction, 1977.

11 Knote/Baraki/Söllner et. al, From requirement to design patterns for ubiquitous computing applications, 1. ten mögliche Ansätze, um das Problem zu lösen, schränken die Kreativität des Anwendenden jedoch nicht ein, da kein vollständiger Lösungsansatz abgebildet wird. ${ }^{12}$ Der Einsatz von Entwurfsmustern hat sich weitgehend etabliert ${ }^{13}$ und ist $\mathrm{zu}$ einem Teil der Ausbildung von Systementwicklern geworden. ${ }^{14}$

\section{Disziplinäres Gestaltungswissen}

Gestaltungswissen, das in den Wissenschaften vom Recht und von der Dienstleistungsqualität erarbeitet wird, muss in die Sprache und die Repräsentationsformen der Systementwickler überführt werden, um Eingang in technischen Systemen zu finden. Gelingt dies nicht, wird es erst einmal von der Systementwicklung ignoriert. Es wird dann allenfalls zu einer Nachentwicklung führen, wenn ein Konflikt um eine spezifische Anforderung eskaliert ist und zu einem Umsatzrückgang oder zu einer rechtlichen Anordnung oder Sanktion geführt hat. Für eine Kodifizierung, die für die Systementwicklung geeignet ist, weisen die Wissenschaften zur Dienstleistungsqualität und zum Recht sehr unterschiedliche Voraussetzungen auf.

\subsection{Gestaltungswissen der Dienstleistungsqualität}

An die Repräsentationsformen der Entwurfskodifizierung kann das Gestaltungswissen zur Dienstleistungsqualität unmittelbar anknüpfen. Es wird in der Regel nicht nur durch natürliche Sprache vermittelt, sondern eine Gestaltungslösung kommt häufig in Ergänzung mit Programmiercode vor. Jedoch sollte diese Verwendungsweise von Gestaltungswissen in der Kodifizierung von Gestaltungsvorschlägen zur Dienstleistungsqualität stärker berücksichtigt werden. Allerdings fehlt Entwurfsmustern zur Dienstleistungsqualität bislang ein interdisziplinärer Bezug, der den Einsatz über eine Disziplin hinaus ermöglichen würde.

\subsection{Gestaltungswissen des Rechts}

Während sich Gestaltungswissen der Dienstleistungsqualität eindeutig zuschreiben lässt, entwickelt die Rechtswissenschaft kaum Wissen, dass sich mit Methoden und Konstruktionen für die Systementwicklung beschäftigt. Allerdings hat die Bedeutung von rechtlichem Wissen in der Systementwicklung in den letzten Jahrzehnten enorm zugenommen. Je stärker Informationstechnik das Arbeiten, Wirtschaften und Verwalten prägt, desto umfangreicher müssen informationstechnische Systeme rechtliche Anforderungen berücksichtigen.

Dies gilt insbesondere für das Datenschutzrecht. In einer Welt, die intensiv durch Informationstechnik geprägt ist, wird sich Recht nur durchsetzen können, wenn es auch Eingang in die Gestaltung von Techniksystemen findet. ${ }^{15}$ Dementsprechend fordert Art. 25 Abs. 1 DSGVO Datenschutz durch Systemgestaltung. Danach müssen bereits in der Entwicklung der Technik

$12 \mathrm{Gamma} / \mathrm{Helm} / \mathrm{Johnson}$ et al., Design Patterns. Elements of Reusable Object-Oriented Software, 1994.Gamma/Helm, Richard, Johnson, Ralph/Vlissides, AddisonWesley Professional 1994.

13 Borchers, Teaching $\mathrm{HCl}$ design patterns: Experience from two university courses, $\mathrm{CHI} 2002$ international conference on human factors of computing systems 2002.

14 Kostas/Koukouletsos/Dearden et al., Teaching Usability Principles with Patterns and Guidelines, 159.

15 Siehe Roßnagel, MMR 2020, $222 \mathrm{ff}$. 
datenschutzfreundliche Kriterien berücksichtigt werden. ${ }^{16}$ Das rechtliche Gestaltungswissen unterscheidet sich jedoch grundsätzlich von technischem Gestaltungswissen. So stellt beispielsweise Art. 25 DSGVO nur wenig konkrete Maßnahmen für die Umsetzung der Norm in der Praxis bereit, was den Verantwortlichen einen großen Spielraum in der Anwendung der Norm ermöglicht. ${ }^{17}$ Dieser Spielraum kann auf der einen Seite eine individuelle Umsetzung ohne starre Vorgaben ermöglichen. Andererseits bleibt immer Unsicherheit bestehen, inwieweit die Norm zufriedenstellend umgesetzt wurde oder die Umsetzung vielleicht sogar zu einer Sanktion führt.

Das Recht enthält kein explizites Gestaltungswissen für Techniksysteme, obwohl es gerade im Datenschutzrecht viele Anforderungen an bestimmte technische Funktionen stellt. Nur selten enthält es einen direkten Bezug zur tatsächlichen Umsetzung seiner Anforderungen. Dies erschwert die Durchsetzungsfähigkeit des Rechts gegenüber der Technik enorm. ${ }^{18}$ Außerdem sind die Anforderungen des Rechts, im Gegensatz zum Gestaltungswissen der Systementwicklung, in Fließtext formuliert und müssen für die Anwendung durch den Systementwickler interpretiert werden. Dies führt meist dazu, dass sie ignoriert werden.

\section{Ansätze für die interdisziplinäre Zusammenarbeit}

Das Zusammenspiel zwischen Recht und Technik soll dahingehend verbessert werden, dass beide gemeinsam rechtsverträgliche Systeme entwickeln können. Auf der einen Seite muss das Recht neue Instrumente entwickeln, um mit anderen Disziplinen agieren zu können. Auf der anderen Seite muss sich die Systementwicklung auf das Recht einlassen und fremdes Fachwissen in bestehende Abläufe integrieren.

Die Systementwicklung hat bereits einige Ansätze entwickelt, die interdisziplinäre Zusammenarbeit zu unterstützen. So helfen Prototypen bereits früh im Entwicklungsprozess dabei, einen Eindruck von dem Endprodukt zu bekommen und je nach Detailgrad des Prototyps bereits erste Funktionalitäten erproben zu können. Um Entwickler noch früher im Entwicklungsprozess unterstützen zu können, haben sich Entwurfsmuster in der Ausbildung von Entwicklern etabliert. Entwurfsmuster repräsentieren Lösungen für immer wieder auftretende Probleme. Ein Entwurfsmuster definiert also die Grundstruktur einer Lösung für ein spezifisches Problem, beschreibt aber noch keine vollständige Lösung des Problems. Dieser Ansatz unterstützt den Entwickler bei der Lösung des Problems, ohne die Kreativität einzuschränken.

\section{Interdisziplinäre Entwurfsmuster}

Um dem Problem von Konflikten zwischen rechtlichen und Anforderungen der Dienstleistungsqualität entgegenzuwirken, ist eine gemeinsame Sprache zwischen Entwicklern und Rechtsex-

16 Hansen, in: Simitis/Hornung/Spiecker, Datenschutzrecht, 2019, Art. 25, Rn. $28 \mathrm{ff.}$; Thies/Knote/Jandt et al., Anforderungs- und Entwurfsmuster als Instrumente des Privacy by Design, in: Roßnagel/Friedewald/Hansen, Die Fortentwicklung des Datenschutzes 2018, 175

17 Knote/Thies/Söllner et al., Informatik Spektrum, 2020, 118 (121).

18 Siehe näher Roßnagel, MMR 2020, 222 (226f.). perten in der Form von interdisziplinären Entwurfsmustern zu erproben. Die Entwurfsmuster sollen beiden Disziplinen ermöglichen, das Wissen der Disziplinen zu integrieren. Dazu wurde im DFG-Forschungsprojekt „Anforderungs- und Entwurfsmuster zur rechtsverträglichen und qualitätszentrierten Entwicklung kontextsensitiver Applikationen“" (AnEkA) der aus der Systementwicklung bekannte Ansatz von Entwurfsmustern für wiederkehrende Probleme verwendet und mit rechtlichem Gestaltungswissen kombiniert.

\subsection{Entwicklung der Entwurfsmuster}

Als Grundlage für die Entwicklung der Entwurfsmuster dienen Konflikte zwischen Anforderungen des Rechts und der Dienstleistungsqualität, wie sie in Anforderungsmustern festgehalten sind. ${ }^{19}$ Anforderungsmuster lösen noch keine konkreten Umsetzungsprobleme im Entwicklungsprozess, sondern dienen zunächst als Unterstützung bei der Spezifikation von Anforderungen. Daher werden für die Umsetzung der Anforderungen in der Praxis konkrete Muster benötigt, um widersprüchliche Anforderungen an das System zu lösen.

Dafür werden die Ziele der Anforderungsmuster der Rechtsverträglichkeit und der Dienstleistungsqualität gegenübergestellt, um mögliche Zusammenhänge und Konflikte zu identifizieren und um auf der Grundlage konfliktärer Anforderungsmuster Entwurfsmuster zu entwickeln. Jedes Anforderungsmuster enthält Informationen über die Priorisierung im Entwicklungsprozess. Es wird ein dreistufiges Verfahren zur Priorisierung der Relevanz, beginnend mit niedriger Priorität über mittlere bis hohe Priorität, verwendet. Dieses Verfahren ermöglicht eine Auswahl der erforderlichen Entwurfsmuster zu treffen. So erfordern nur Konflikte zwischen Anforderungsmustern, die beide eine hohe Priorität haben, ein Entwurfsmuster.

Um kreative Lösungen für die Konflikte zu finden, die zwischen Rechtsverträglichkeit und Dienstleistungsqualität entstehen, wurden verschiedene Methoden wie Workshops und Experteninterviews durchgeführt. Dabei wurde stets dafür gesorgt, dass sowohl Rechtsexperten als auch Systementwickler einbezogen wurden, um sicherzustellen, dass die Lösung beide Disziplinen zufriedenstellend abdeckt. Dieser Ansatz ermöglicht es, vorhandenes Wissen und bekannte Lösungsansätze für immer wieder auftretende Probleme zu nutzen und in die interdisziplinären Entwurfsmuster zu integrieren.

\subsection{Aufbau der Entwurfsmuster}

Die interdisziplinären Entwurfsmuster sollen einen möglichst großen Mehrwert für beide Disziplinen haben und insbesondere die Entwickler so unterstützen, dass diese durch die Anwendung der Entwurfsmuster grundlegendes Rechtswissen aufbauen und nicht immer wieder auf die Entwurfsmuster zugreifen müssen. Deshalb wurde bei der Gestaltung der Entwurfsmuster nicht der, aus der Systementwicklung bekannte Aufbau für Entwurfsmuster, verwendet, sondern ein Aufbau basierend auf verschiedenen Theorien und Erkenntnissen der Kodifizierung von Gestaltungswissen und die kognitive Last entwickelt.

Jedes Entwurfsmuster wird in einer einseitigen Darstellung präsentiert, wodurch alle notwendigen Informationen auf einen

\footnotetext{
19 Siehe Dickhaut/Janson/Roßnagel/Leimeister, in diesem Heft.
} 
Abbildung 1 | Beispielhaftes Entwurfsmuster „Datenschutzfreundliches Nutzerprofil

\begin{tabular}{|c|c|c|c|c|c|}
\hline \multirow{2}{*}{\multicolumn{2}{|c|}{ Datenschutzfreundliches Nutzerprofil }} & \multicolumn{3}{|c|}{ Zeitpunkt im Entwicklungsprozess } & \\
\hline & & \multicolumn{2}{|c|}{$\begin{array}{l}\underline{X} \text { Interaktionsmuster } \\
\square \text { Lernmuster }\end{array}$} & $\begin{array}{l}\square \text { Architekturmuster } \\
\square \text { Datenverarbeitungsrmuster }\end{array}$ & \\
\hline \multicolumn{5}{|c|}{$\begin{array}{l}\text { Ziel } \\
\text { Das Profil des Nutzers enthält besonderen Kategorien personenbezogener Daten. Auch werden im Nutzungsverlauf keine } \\
\text { Inferenzen auf besondere Kategorien personenbezogener Daten gebildet und im Nutzerprofil abgelegt. }\end{array}$} & \\
\hline $\begin{array}{l}\text { Rec } \\
\text { Differenzierte Venvendungszweck } \\
\text { Nichtverkettbarkeit } \\
\text { Vermeidung personenbezogener }\end{array}$ & $\begin{array}{l}\text { Anforc } \\
\text { Nachwollziehbare Darstellung } \\
\text { der Datenverarbeitung } \\
\text { Kein vollständiges Nutzerprafil }\end{array}$ & $\begin{array}{l}\text { rungen } \\
\text { : Erklär } \\
\text { - Reme } \\
\text { Bekan }\end{array}$ & \multicolumn{2}{|c|}{$\begin{array}{l}\text { Dienstleistungsqualität } \\
\text { atenschutz } \\
\text { estaltung }\end{array}$} & \\
\hline $\begin{array}{l}\text { Recht } \\
\text { - Löschung und Berichtigung von } \\
\text { Nutzerdaten } \\
\text { - Information des Nutzers }\end{array}$ & $\begin{array}{l}\text { Dienstleistungsqualit } \\
\text { - Keine Transparenz für den N } \\
\text { System unpassende Antwort } \\
\text { - Hoher Implementierungsauf } \\
\text { Individualisierungsgrad des } \\
\text { steuerbar }\end{array}$ & \begin{tabular}{l|} 
\\
tzer, wenn \\
ingt \\
and \\
stems
\end{tabular} & $\begin{array}{l}\text { - Empathi } \\
\text { - Schutzd } \\
\text { - } \text { Kein volls } \\
\text { - } \text { Keinspar }\end{array}$ & $\begin{array}{l}\text { nflüsse } \\
\text { benssphäre } \\
\text { rofil } \\
\text { Jerer Kategorien von Daten }\end{array}$ & \\
\hline \multicolumn{6}{|c|}{$\begin{array}{l}\text { - Spezifikation der benötigten Daten zur Profilbildung } \rightarrow \text { konkreter Verarbeitungszweck } \\
\text { Generell keine Verarbeitung besonderer Kategorien von Daten (Rasse, ethnische Herkunft, politische Meinungen, religiöse oder weltanschauliche } \\
\text { Uberzeugungen, Gewerkschaftszugehörigkeit, genetische Daten, biometrische Daten, Gesundheitsdaten, Daten zum Sexualleben oder zur sexuelen } \\
\text { Orientierung) } \\
\text { - Nur Verarbeitung solcher Daten, die zur Diensterbringung unbedingt erforderlich sind } \\
\text { - Transparenz über alle Datenverarbeitungsvorgänge } \rightarrow \text { Entwurfsmuster Berechtigungsmanagement } \\
\text { - Möglichkeit einer digitalen Selbstauskuntt } \\
\text { - Regelmäßige Öberprüfung hins. Einhaltung des Verarbeitungszweck, ggf. } \rightarrow \text { Entwurfsmuster Löschroutinen }\end{array}$} \\
\hline \multicolumn{5}{|c|}{ 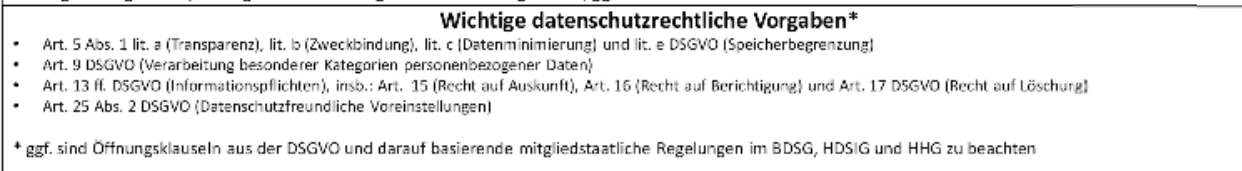 } & \\
\hline Bestätigung der Umsetzung de & des Entwurfsmusters & Datum & & Unterschrift & \\
\hline
\end{tabular}

Da jedes Entwurfsmuster eine Lösung darstellt, die Anforderungen sowohl des Rechts als auch der Dienstleistungsqualität umsetzt, werden auch Konsequenzen für beide Disziplinen dargestellt, die durch die Anwendung des Entwurfsmusters möglicherweise auftreten können. Beispielhafte Konsequenzen in dem Entwurfsmuster „Datenschutzfreundliches Nutzerprofil" sind auf Seiten der Rechtsverträglichkeit „Löschung und Berichtigung von Nutzerdaten" und "Information des Nutzers" sowie auf Seiten der Dienstleistungsqualität „hoher Implementierungsaufwand“. Die dargestellten Konsequenzen können positiv, aber auch negativ sein. Im Kern des Entwurfsmusters steht die

Blick verfügbar sind und nicht viele verschiedene Seiten gelesen werden müssen, um die gesuchte Information finden zu können. Abbildung 1 zeigt ein beispielhaftes Entwurfsmuster.

Für die komprimierte Darstellung aller Informationen muss der Inhalt beider Disziplinen auf die notwendigsten Informationen gekürzt werden, ohne dass daraus falsche Interpretationen des Inhalts resultieren. Als Lösung wurden einzelne Kategorien gebildet, die eine übersichtliche Einheit der einzelnen Themenschwerpunkte repräsentieren und alle notwendigen Informationen vereinen. Diese Kategorien sind: Zeitpunkt im Entwicklungsprozess, Ziel, Anforderungen, Konsequenzen, Einflüsse, Lösung und wichtige datenschutzrechtliche Vorgaben. Einige der Kategorien werden nochmals in Rechtsverträglichkeit und Dienstleistungsqualität aufgeteilt. Die sieben Kategorien werden durch einen eindeutigen Namen des Entwurfsmusters und ein Feld, in dem die Anwendung des Entwurfsmusters mit einem Datum und einer Unterschrift bestätigt werden kann, ergänzt. Der eindeutige Name des Entwurfsmusters repräsentiert den Inhalt des Musters und impliziert somit auch das Ziel der Anwendung des Musters. Dies ermöglicht den Austausch über konkrete Entwurfsmuster, ohne dabei den Inhalt genauer erklären zu müssen.

Jedes Entwurfsmuster hat eine Beschreibung des Ziels der Umsetzung des Musters, die in ein bis zwei Sätzen den idealen Zielzustand nach der Umsetzung beschreibt. Ergänzt wird die Beschreibung des Ziels durch die Anforderungen, die in beiden Disziplinen als Grundlage für die Erstellung des Entwurfsmusters gedient haben. Diese werden in der Darstellung des Musters jeweils für Rechtsverträglichkeit und Dienstleistungsqualität aufgelistet. Neben den Anforderungen gibt es die Kategorie „Einflüsse“, die Kriterien beinhaltet, die als Grundlage der Entwurfsmuster verwendet werden können.
Lösung, die Lösungsansätze für immer wieder auftretende Probleme darstellt. Die Lösung wird stichpunktartig dargestellt und verbindet so Gestaltungswissen des Rechts und der Systementwicklung. Auch die Art wie das Wissen beider Disziplinen kodifiziert wird, berücksichtigt die Unterschiede der einzelnen Disziplinen. In der Lösung können Verweise auf andere Entwurfsmuster vorkommen, die bei der Anwendung helfen sollen, eine ganzheitliche interdisziplinäre Lösung des Systems zu entwickeln und nicht nur in einzelnen Elementen sowohl Rechtsverträglichkeit als auch Dienstleistungsqualität umzusetzen. Neben den bisher dargelegten Kategorien, die beide Disziplinen vereinen, repräsentieren die Kategorien „Zeitpunkt im Entwicklungsprozess“ und „Wichtige datenschutzrechtliche Vorgaben“ primär eine Disziplin. In der Kategorie zu dem Zeitpunkt im Entwicklungsprozess werden Informationen bereitgestellt, die eine Aussage darüber geben, an welchem Prozess das dargestellte Entwurfsmuster anknüpft. Aus rechtlicher Perspektive werden in der Kategorie „Wichtige datenschutzrechtliche Vorgaben“ datenschutzrechtliche Normen, die bei der Umsetzung des jeweiligen Entwurfsmusters adressiert werden, angegeben. Diese Auflistung dient lediglich dazu, einen schnellen Überblick über möglicherweise relevante datenschutzrechtliche Vorgaben zu geben. Sie erfasst SPA in genereller Hinsicht, ist daher notwendigerweise sehr abstrakt und ersetzt nicht die rechtliche Prüfung der zu erfüllenden rechtlichen Vorgaben im Einzelfall.

Die dargestellten Kategorien werden im unteren Bereich des Entwurfsmusters durch ein Feld ergänzt, das ermöglicht, in der Systementwicklung zu dokumentieren, dass das Entwurfsmuster so wie es dargestellt wird, umgesetzt wurde. 


\section{Anwendung der Entwurfsmuster}

Mit Hilfe des interdisziplinären Musterkatalogs wurde ein KIbasierter Lernassistent entwickelt, der mit Studierenden in einer Grundlagenlehrveranstaltung für die Vorbereitung auf eine Klausur eingesetzt und evaluiert wurde..$^{20}$ An der Entwicklung waren drei Entwickler beteiligt, die über praktische Erfahrung in der Systementwicklung verfügen. Für die Entwicklung wurden sie aufgefordert, den Musterkatalog zu verwenden und auf der Grundlage der Lösungsansätze in den Entwurfsmustern vorzugehen.

\subsection{Anforderungen Lernassistent}

Wichtige Anforderungen auf Seiten der Dienstleistungsqualität an den smarten persönlichen Lernassistenten basierten auf den Anforderungen „Personalisierung“ und „Lernfähigkeit“. So wäre es aus Qualitätssicht wünschenswert, dass der Lernassistent den Nutzer nach erstmaliger Nutzung wiedererkennen kann. Sowie, dass der Lernassistent auf Basis der Bewertungen des Nutzers mit fortschreitender Nutzungsdauer Informationen, die auf den Nutzer angepasst sind, ausgeben kann.

Die rechtlichen Anforderungen „Datensparsamkeit und Datenvermeidung“, „Kernbereichsschutz“ und „Keine Profilbildung“ verlangen vom Lernassistenten hingegen, dass nur solche personenbezogenen Daten verarbeitet werden, die zur Erreichung des Verarbeitungszwecks erforderlich sind, und dass er kein umfassendes Persönlichkeitsprofil des Nutzers erstellt.

Um diese Zielkonflikte aufzulösen und zufriedenstellenden Lösungen zuzuführen, kommen die interdisziplinären Entwurfsmuster zum Einsatz, die die konfliktären Anforderungen des Rechts und der Dienstleistungsqualität adressieren und mögliche Lösungswege aufzeigen.

\subsection{Lösungen in Entwurfsmustern}

Die Anforderungen der „Personalisierung“ und „Datensparsamkeit und Datenvermeidung "lassen sich nicht gleichermaßen umsetzen, da sie einander widersprechen. Würde man alle Anforderungen der Dienstleistungsqualität voll verwirklichen, würde dies gegen rechtliche Anforderungen verstoßen. Würden alle rechtlichen Anforderungen ohne Rücksicht auf andere Anforderungen umgesetzt, würde hingegen die Dienstleistungsqualität des Lernassistenten leiden. Um die beschriebenen Konflikte bestmöglich aufzulösen und einer qualitativ hochwertigen sowie rechtsverträglichen Lösung zuzuführen, wurde das Entwurfsmuster „Datenschutzfreundliches Nutzerprofil“ genutzt.

Das Entwurfsmuster „Datenschutzfreundliches Nutzerprofil“ "löst den beschriebenen grundlegenden Zielkonflikt, indem es spezifiziert, welche personenbezogenen Daten des Nutzers verarbeitet und im Nutzerprofil gespeichert werden dürfen. Dabei

20 Nach Knote/Janson/Söllner et al., Journal of the Association for Information Systems (JAIS), 2020 (für Veröffentlichung akzeptiert) ein sog. "General Activity Assistant" für den Bereich des digitalen Lernens. wird in der Lösung unter anderem vorgeschlagen, dass besondere Kategorien personenbezogener Daten nicht zur Profilbildung genutzt werden sollten. Eine strenge Einhaltung des Zweckbindungsgrundsatzes stellt sicher, dass nur solche personenbezogenen Daten verarbeitet werden, die zur Zweckerreichung erforderlich sind. Gleichzeitig garantiert diese Lösung auch, dass die Anforderungen auf Seiten der Dienstleistungsqualität umgesetzt werden können. Eine personalisierte Art der Dienstleistungserbringung wird ermöglicht, wenn es sich nicht um Daten, die zu besonderen Kategorien gehören, handelt. Um dabei die informationelle Selbstbestimmung des Nutzers zu gewährleisten, erfolgt eine Personalisierung nur, wenn der Nutzer aktiv einwilligt. Ohne Einwilligung durch den Nutzer, wird kein Nutzerprofil angelegt. Außerdem wird im Entwurfsmuster vorgeschlagen, eine Identifizierung des Nutzers auf dem Endgerät auf Basis eines selbstgewählten Pseudonyms zu ermöglichen. Durch die Pseudonymisierung wird kein Nutzerprofil angelegt, das unmittelbar Rückschlüsse auf die Person selbst zulässt. Dennoch wird so erreicht, dass der Nutzer eine Möglichkeit zur Identifizierung hat und der Lernassistent dadurch immer wieder auf den aktuellen Lernfortschritt zugreifen kann, um die Lehrinhalte daran anzupassen.

Des Weiteren besagt das Entwurfsmuster, dass der Verwendungszweck der Datenverarbeitung präzise und differenziert angegeben werden muss und eine granulare Einwilligungsmöglichkeit dem Nutzer ermöglichen soll, nicht allen Punkten der Datenverarbeitung zuzustimmen.

\section{Abschließende Bewertung}

Entwurfsmuster können eine effektive Methode darstellen, die Systementwicklung zu unterstützen, sodass qualitativ hochwertige und datenschutzgerechte Systeme entwickelt werden können. Die Möglichkeit, durch interdisziplinäre Entwurfsmuster auf der einen Seite die Systementwicklung für Rechtsexperten greifbar zu machen und auf der anderen Seite Entwickler im Verständnis von rechtlichen Anforderungen an die Systementwicklung zu unterstützen, bietet die Chance einer „gemeinsamen Sprache“. Der Einsatz der entwickelten Entwurfsmuster hat sich bereits in diversen Studien als erfolgreich erwiesen. ${ }^{21}$ Ein wichtiges Merkmal für die Entwicklung von interdisziplinären Entwurfsmustern ist die aktive Integration aller Disziplinen. Nur wenn die Entwurfsmuster die Besonderheiten des Gestaltungswissens der einzelnen Disziplinen berücksichtigt, können sie im Kern der Lösung eine interdisziplinäre Verbindung repräsentieren. Durch ihren expliziten und konkreten Technikbezug können interdisziplinäre Entwurfsmuster einen wichtigen Beitrag dazu leisten, die abstrakten Vorgaben der DSGVO auch für Systementwickler verständlich und umsetzbar darzustellen und eine Brücke zwischen Recht und Systementwicklung zu bilden.

21 Für Veröffentlichung akzeptiert: Dickhaut/Janson/Leimeister, Design Science Research in Information Systems and Technology (DESRIST), 2020. 